\title{
ANTIBACTERIAL ACTIVITY AND GREENER METHOD OF SYNTHESIS OF HETEROCYCLIC COMPOUNDS INCORPORATING BENZOTHIAZOLE MOIETY USING IONIC LIQUID AS SOLVENT
}

\author{
Madhu Toshniwal ${ }^{1,2, *}$, Mahendra Bundel ${ }^{1}$, Prashant Kriplani ${ }^{2}$, Ravikant ${ }^{3}$, \\ Rashmi Pareek ${ }^{4}$ and Arun Pareek ${ }^{1}$ \\ ${ }^{1}$ Analytical \& Pharmaceutical Research Laboratory, Department of Chemistry, S. P. C. \\ Government College Ajmer, 305001(Rajasthan) India. \\ ${ }^{2}$ Government Women Engineering College Ajmer, 305002(Rajasthan) India. \\ ${ }^{3}$ Helix BioGenesis Lab, Noida,(U.P.) India. \\ ${ }^{4}$ Government College, Behror. (Rajasthan) India \\ *E-mail: madhutoshniwal@gweca.ac.in
}

ABSTRACT

A novel series of 2-amino-6-substituted benzothiazoles with halo esters and halo ketones have been synthesized by a conventional method. The same series was also synthesized under Microwave condition using ionic liquids. The yield of products by Microwave synthesis with ionic liquids is much better than the conventional method and also save time and energy. All the reported compounds in this article have been characterized by IR, ${ }^{1} \mathrm{H}$ NMR data and elemental analysis in full accordance with their expected (depicted) structures. The reported compounds were further screened for their antibacterial activity using Ampicillin as standard drug. The compounds showed excellent activity against bacteria Pseudomonas Aeruginosa, Klebsiella pneumonia and Staphylococcus aureus.

Keywords: Benzothiazole, Halo Esters, Halo Ketones, Ionic-Liquids (Green Solvent), Microwave Irradiation, Antibacterial

(c) RASĀYAN. All rights reserved

\section{INTRODUCTION}

Heterocyclic compounds are useful candidates from a medicinal point of view. Derivatives of benzothiazole are useful as a starting material in many bioactive structures. Several benzothiazole derivatives have been used against anti-proliferative and DNA binding properties ${ }^{1}$, anticancer and antioxidant activity against pancreatic cancer cell $^{2}$, antitumor and antiviral ${ }^{3}$, anti infective ${ }^{4}$, Human MCF7cancer cell line ${ }^{5}$ Novel non sulfamide NEDD8 activating enzyme inhibitors ${ }^{6}$, antidepressant ${ }^{7}$, hypoglycaemic and hypolipidemic ${ }^{8}$, cervical cancer ${ }^{9}$, anticonvulsant ${ }^{10}$, anti-acetyl cholinesterase ${ }^{11}$, kinase inhibitor $^{12}$, anti tuberculotic ${ }^{13}$. Looking at the importance of this nuclei, we have synthesized new benzothiazole derivatives by conventional as well as microwave irradiation technique. The traditional method of synthesis has some disadvantages like typical optimization, low yield, low reaction time and inconvenience of handling. Use of ionic liquids in this context has been proven beneficial ${ }^{14}$. The important properties of ionic liquids are their thermal stability, non-measurable vapor pressure, solvating properties and wide liquid range for different materials. Another notable characteristic of ionic liquids is there: miscibility with water or organic solvents and designability can be managed through the chain lengths of the cations and by choosing different anions. Because the properties of ionic liquids they attract great attention in many areas including physical chemistry, organic chemistry, electrochemistry and in the various field of engineering. As their non-volatility has a low impact on human health and the environment, they are important for "green chemistry".

Rasayan J. Chem., 12(3), 1294-1297(2019)

http://dx.doi.org/10.31788/RJC.2019.1235207

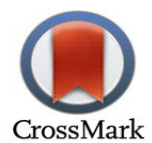


Molten ionic liquid-TBAB tetra butyl ammonium bromide) can be used as an efficient, green and novel media $^{15}$ in organic synthesis. It is a recyclable catalyst for the synthesis under conventional and microwave heating in excellent yields ${ }^{16}$. All the reported derivatives of benzothiazole were screened by using the standard drug for their antibacterial activity.

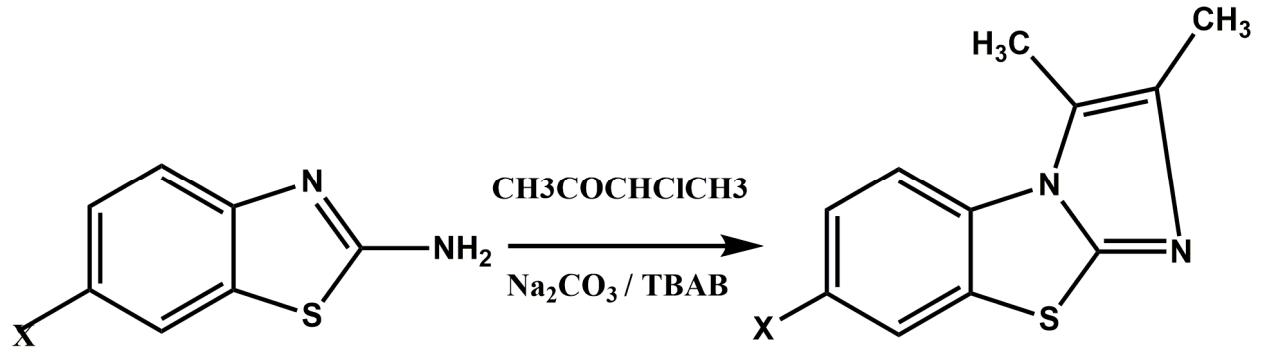

Scheme-1: Synthesis of 7-Substituted-2, 3-dimethylimidazo [1,3]benzothiazole

\section{Methods and Materials}

\section{EXPERIMENTAL}

Chemicals were used without further purification. The melting points are uncorrected and were taken in open capillaries. The completion of reaction and purity of reported compounds were checked by TLC (thin layer chromatographic). FTIR Perkin Elmer (Spectrum 2) Spectrophotometer $\left(\mu\right.$ in $\mathrm{cm}^{-1}$ ) were used for IR spectra by using $\mathrm{KBr}$ disc. ${ }^{1} \mathrm{H}$ NMR was recorded in DMSO and $\mathrm{CDCl}_{3}$ with tetramethylsilane (TMS) as an internal standard at $400 \mathrm{MHz}$ on an ECS (JEOL) NMR Spectrophotometer. The Chemical shift was measured in parts per million (ppm). Fast atom bombardment mass spectra (FABMS) were recorded at room temperature on a JEOL SX-102/DA-6000 Mass Spectrophotometer using Argon/Xenon $(6 \mathrm{kV}, 10 \mathrm{~mA})$ as the FAB gas. The accelerating potential was $10 \mathrm{kV}$. Elementar Vario EL III Carlo Erba1108 elemental analyzer was used for elemental analysis.

\section{Method used for the Synthesis of 7/5-Substituted (F, Cl, $\mathrm{Br}, \mathrm{OCH}_{3}, \mathrm{NO}_{2}$ )-2, 3-dimethylimidazo [1, 3] benzothiazole}

A mixture of 0.01 mole of 2-amino-4/6- Substituted benzothiazole and 0.01 mole of halo ketone were refluxed in $20 \mathrm{ml}$ of ethanol. The same reaction was carried out in a microwave reactor (CEM Discover) using $\mathrm{Na}_{2} \mathrm{CO}_{3}$ and $\mathrm{TBAB}$ (tetra butyl ammonium bromide) as an ionic liquid. TLC (thin layer chromatographic) method was used for the monitoring of reaction (Hexane: acetone 60:40). After reaction completion, the mixture was allowed to cool down and attain room temperature then solid separated was filtered. Redistilled ethanol was used for crude product in recrystallized from. TBAB is recovered.

\section{Spectral Analysis of Compounds (a-e)}

\section{a. 7-Chloro-2, 3-dimethylimidazo $\{2,1-b\}[1,3]$ benzothiazole} IR $\left(\mathrm{cm}^{-1}\right)$ : 1305(C-S), 1560(C=N), 856(C-Cl), $\left.1110(\mathrm{C}-\mathrm{N}),\right) ;{ }^{1} \mathrm{H}$ NMR (400 MHz): 1.64(s, 6H, $\left.\mathrm{CH}_{3}\right)$, 7.01-7.39 (m, 3H, Ar-H); EI-MS m/z: 236.72

b. 7-Bromo-2, 3-dimethylimidazo $\{2,1-b\}[1,3]$ benzothiazole IR $\left(\mathrm{cm}^{-1}\right): 1304(\mathrm{C}-\mathrm{S}), 1529(\mathrm{C}=\mathrm{N}), 810(\mathrm{C}-\mathrm{Br}) 1103(\mathrm{C}-\mathrm{N}) ;{ }^{1} \mathrm{H}$ NMR $(400 \mathrm{MHz}): 2.1\left(\mathrm{~s}, 6 \mathrm{H}, \mathrm{CH}_{3}\right)$, 6.90-7.14 (m, 3H, Ar-H); EI-MS m/z:281.17

c. 7-Nitro-2, 3-dimethylimidazo $\{2,1-b\}[1,3]$ benzothiazole IR $\left(\mathrm{cm}^{-1}\right): 1319(\mathrm{C}-\mathrm{S}), 1580(\mathrm{C}=\mathrm{N}), 1520\left(\mathrm{NO}_{2}\right), 1119(\mathrm{C}-\mathrm{N}) ;{ }^{1} \mathrm{H}$ NMR $(400 \mathrm{MHz}): 2.2\left(\mathrm{~s}, 6 \mathrm{H}, \mathrm{CH}_{3}\right)$, 7.10-7.42 (m, 3H, Ar-H); EI-MS m/z: 247.27

d. 7-Ehoxy-2, 3-dimethylimidazo $\{2,1-b\}[1,3]$ benzothiazole IR $\left(\mathrm{cm}^{-1}\right): \quad 1325(\mathrm{C}-\mathrm{S}), \quad 1068(\mathrm{C}-\mathrm{O}-\mathrm{C} \quad$ sym. $), \quad 1111(\mathrm{C}-\mathrm{N}), \quad 1270(\mathrm{C}-\mathrm{O}-\mathrm{C}$ asym. $), \quad 1625(\mathrm{C}=\mathrm{N})$; ${ }^{1} \mathrm{H}$ NMR(400 MHz): 6.90-7.21 (m, 3H, Ar-H), 1.78(s, 6H, $\left.\mathrm{CH}_{3}\right), 3.32\left(\mathrm{q}, 2 \mathrm{H}, \mathrm{CH}_{2}\right), 2.98\left(\mathrm{t}, 3 \mathrm{H}, \mathrm{CH}_{3}\right)$; EI-MS m/z: 247.27

e. 5-Methyl-2, 3-dimethylimidazo $\{2,1-b\}[1,3]$ benzothiazole IR $\left(\mathrm{cm}^{-1}\right): 1536(\mathrm{C}=\mathrm{N}), 1324(\mathrm{C}-\mathrm{S}), 1106(\mathrm{C}-\mathrm{N}), ;{ }^{1} \mathrm{H}$ NMR (400 MHz): 2.53 (s, 3H, $\left.\mathrm{Ar}_{-} \mathrm{CH}_{3}\right), 6.99-7.30$ (m, 3H, Ar-H), 2.1(s, 6H, $\left.\mathrm{CH}_{3}\right)$; EI-MS m/z: 217.28 
RASĀYAN J. Chem.

Vol. 12 | No. 3 |1294 - 1297| July - September | 2019

\section{RESULTS AND DISCUSSION}

The 2-amino-6-substituted benzothiazole was prepared by methods reported in literature ${ }^{17}$. The synthesis of compounds 7/5-Substituted ( $\left.\mathrm{F}, \mathrm{Cl}, \mathrm{Br}, \mathrm{OCH}_{3}, \mathrm{NO}_{2}\right)$-2, 3-dimethylimidazo $\{2,1-\mathrm{b}\}[1,3]$ benzothiazole starting from 2-amino-6-substituted benzothiazole carried out under microwave conditions as well as by conventional method. The reaction was completed within 10-20 minutes using microwave irradiations while the reaction takes 6-12 hours in conventional methods, and yield has also been improved. All the reported compounds have been established on the basis of their spectral and Physico-chemical data (Table-1).

Table-1: Physico-Chemical Data of the Synthesized Compounds (C. M. = Conventional Method, M. W. = Microwave Method)

\begin{tabular}{c|c|c|c|c|c|c}
\hline Drug & $\begin{array}{c}\text { Time } \\
\text { taken } \\
\text { C.M. } \\
(\mathrm{hrs})\end{array}$ & $\begin{array}{c}\text { Time } \\
\text { taken } \\
\text { M.W } \\
(\mathrm{min})\end{array}$ & $\begin{array}{c}\text { Yield } \\
\text { C.M. } \\
\%\end{array}$ & $\begin{array}{c}\text { Yield } \\
\text { M.W. } \\
\%\end{array}$ & $\begin{array}{c}\text { M.P. } \\
\left({ }^{\circ} \mathrm{C}\right) \\
\text { M.W(C.M. })\end{array}$ & $\begin{array}{c}\text { Elemental Analysis } \\
\text { Calculated (Found)\% } \\
\mathrm{H}\end{array}$ \\
\hline $\mathrm{a}$ & 8 & 15 & 50 & 70 & $135(134)$ & $\begin{array}{c}55.81(55.79) 3.83(3.82) 11.83 \\
(11.84) 13.54(13.56) 14.98(14.97)[\mathrm{Cl}]\end{array}$ \\
\hline $\mathrm{b}$ & 7 & 10 & 45 & 75 & $130(128)$ & $\begin{array}{c}46.99(46.98) 3.23(3.24) 9.96(9.95) 11.40 \\
(11.42) 28.42(28.41)[\mathrm{Br}]\end{array}$ \\
\hline $\mathrm{c}$ & 12 & 20 & 35 & 45 & $190(189)$ & $\begin{array}{c}53.43(53.45) 3.67(3.68) 16.99 \\
(16.98) 12.97(12.99) 12.94(12.91)[\mathrm{O}]\end{array}$ \\
\hline $\mathrm{d}$ & 8 & 15 & 60 & 85 & $210(210)$ & $\begin{array}{c}63.39(63.35) 5.73(5.74) \\
11.37(10.97) 13.02(12.87) \\
6.49(6.44)[\mathrm{O}]\end{array}$ \\
\hline $\mathrm{e}$ & 6 & 15 & 65 & 85 & $225(224)$ & $\begin{array}{c}66.63(66.59) 5.59(5.60) 12.95(12.88) \\
14.82(14.84)\end{array}$ \\
\hline
\end{tabular}

\section{Antibacterial Activity}

The antibacterial activity of all the reported compounds was tested in-vitro against Staphylococcus aureus, Pseudomonas aeruginosa and Klebsiela pneumonia by disc diffusion method ${ }^{18}$. The activity was classified as 'slightly active' (diameter $=6-10$ ), "moderately active" (diameter $=10-14 \mathrm{~mm}$ ) and 'highly active' (diameter $>14 \mathrm{~mm}$ ). The diameter of less than $6 \mathrm{~mm}$ was regarded as inactive.

Table 2: Antibacterial Activity of Synthesized Compounds

\begin{tabular}{c|c|c|c|c|c}
\hline S. No. & Compounds & Control & $\begin{array}{c}\text { Pseudomonas } \\
\text { Aeruginosa }\end{array}$ & $\begin{array}{c}\text { Staphylococcus } \\
\text { aureus }\end{array}$ & $\begin{array}{c}\text { Klebsiela } \\
\text { pneumoniae }\end{array}$ \\
\hline 1 & A & --- & ++ & +++ & ++ \\
\hline 2 & B & --- & ++ & ++ & ++ \\
\hline 3 & C & --- & ++ & +++ & +++ \\
\hline 4 & D & --- & +++ & ++ & +++ \\
\hline 5 & E & --- & ++ & ++ & +++ \\
\hline
\end{tabular}

Data represent zone of inhibition $(\mathrm{mm})$ as follows $-(+++)$ for diameter $>14 \mathrm{~mm},(++)$ for diameter $=10-$ $14 \mathrm{~mm}$, (+) for diameter $=6-10$, DMSO was used as a solvent, Solutions are in $\mu \mathrm{g} / \mathrm{ml}$. Ampicillin as Standard drug was used for comparison. The compound was tested at $100 \mu \mathrm{g} / \mathrm{ml}$ concentration. The results showed that the compound $\mathbf{d}$ was highly active against Pseudomonas aeruginosa, compound a and c were highly active against Staphylococcus aureus and the compound $\mathbf{c}, \mathbf{d}$ and $\mathbf{e}$ were highly active against Klebsiela pneumonia.

\section{CONCLUSION}

We have used microwave irradiation technique and TBAB as ionic liq. in this synthetic scheme and this lead to an increase in the yield of compounds and considerable saving the reaction time and energetically profitable. The result shows that ionic liquid is efficient microwave absorbers and recovered so minimize the problem of waste disposal. Compounds a, $\mathbf{c}, \mathbf{d}$ and e show remarkable antibacterial activity. So it is evident that the reported compounds would be great importance in drug development against bacterial infection. 
RASĀYAN J. Chem.

Vol. 12 | No. 3 |1294 - 1297| July - September | 2019

\section{ACKNOWLEDGMENT}

Authors are thankful to the Principal, Govt. Women Engineering College, Ajmer. Dept. of Chemistry, S. P. C. Govt. College, Ajmer and Dr. Rajkumar Joshi, Department of Chemistry, MNIT, Jaipur for laboratory facilities, we are also expressing thanks to Material Research Centre, MNIT, Jaipur for analytical facilities.

\section{REFERENCES}

1. S. J. Maja Cindric, Anja Harej,Sabine Depauw,Marie-Helena David-Cordonnier, European Journal of Medicinal Chemistry, 136, 468(2017), DOI: 10.1016/j.ejmech.2017.05.014.

2. N. Uremis, M. M. Uremis, F. I. Tolun, M. Ceylan, A. Doganer, A. H. Kurt. Anticancer Res., 37, 6381(2017), DOI: 10.21873/anticanres.12091.

3. T. Akhtar, S. Hameed, N. A. Al-Masoudi, R. Loddo, P. La Colla, Acta Pharm. 58, 135(2008), DOI: 10.2478/v10007-008-0007-2.

4. K. K. B. Prabodh Chander Sharma, Aakash Deep, Meenakshi Pathak, Current Topics in Medicinal Chemistry, 17, 208(2017), DOI:10.2174/1568026616666160530152546.

5. L. W. Mohamed, A. T. Taher, G. S. Rady, M. M. Ali, A. E. Mahmoud, Chem Biol Drug Des., 89, 566(2017), DOI: $10.1111 /$ cbdd.12879.

6. H. Ma, C. Zhuang, X. Xu, J. Li, J. Wang, X. Min, W. Zhang, H. Zhang, Z. Miao, Eur J Med Chem. 133, 174(2017), DOI: 10.1016/j.ejmech.2017.03.076.

7. U. Demir Ozkay, C. Kaya, U. Acar Cevik, O. D. Can, Molecules., 22, (2017), DOI: 10.3390/molecules22091490.

8. A. Ahmadi, M. Khalili, L. Sohrabi, N. Delzendeh, B. Nahri-Niknafs, F. Ansari, Mini. Rev. Med. Chem., 17, 721(2017), DOI: 10.2174/1389557516666161130095407.

9. M. Singh, A. Modi, G. Narayan, S. K. Singh, Anticancer Drugs, 27, 519(2016), DOI: 10.1097/CAD.0000000000000357.

10. D. C. Liu, H. J. Zhang, C. M. Jin, Z. S. Quan, Molecules, 21, 164(2016), DOI: 10.3390/molecules21030164.

11. U. A. Mohsen, Z. A. Kaplancikli, Y. Ozkay, L. Yurttas, Drug Res (Stuttg), 65, 176(2015), DOI: 10.1055/s-0034-1375613.

12. M. Okaniwa, M. Hirose, T. Arita, M. Yabuki, A. Nakamura, T. Takagi, T. Kawamoto, N. Uchiyama, A. Sumita, S. Tsutsumi, T. Tottori, Y. Inui, B. C. Sang, J. Yano, K. Aertgeerts, S. Yoshida, T. Ishikawa, J. Med. Chem., 56, 6478(2013), DOI: 10.1021/jm400778d.

13. V. N. Telvekar, V. K. Bairwa, K. Satardekar, A. Bellubi, Bioorg. Med. Chem. Lett., 22, 649 (2012), DOI: $10.1016 / \mathrm{j} . \mathrm{bmcl} .2011 .10 .064$.

14. O. Algul, A. Kaessler, Y. Apcin, A. Yilmaz, J. Jose, Molecules., 13, 736(2008), DOI: 10.3390/molecules 13040736 .

15. Z. R. Shadpour Mallakpour, European Polymer Journal., 43, 1510(2007), DOI: 10.1016/j.eurpolymj.2007.01.006.

16. M. V. C. Srinivas Kantevari, A.P.Rudra Das,Srinivasu V.N.Vuppalapati,N.Lingaiah, Catalysis Communications, 9, 1575(2008), DOI:10.1016/j.catcom.2008.01.003.

17. T. D. S. Bradshaw, M.F.G., A. D. Westwell, Current Medicinal Chemistry, 8, 203(2001), DOI: 10.2174/0929867013373714.

18. J.-C. F. GinetteSimon-Sylvestre, Advances in Agronomy, 31, 1(1980), DOI:10.1016/S00652113(08)60136-4.

[RJC-5207/2019] 